\title{
Cyclin D3 coordinates the cell cycle during differentiation to regulate erythrocyte size and number
}

\author{
Vijay G. Sankaran, ${ }^{1,2,3,4,17}$ Leif S. Ludwig, ${ }^{2,5,6,17}$ Ewa Sicinska, ${ }^{7,18}$ Jian Xu, ${ }^{8,9,18}$ Daniel E. Bauer, ${ }^{8,9,18}$ \\ Jennifer C. Eng ${ }^{1,2}$ Heide Christine Patterson, ${ }^{2,10,11}$ Ryan A. Metcalf, ${ }^{12}$ Yasodha Natkunam, ${ }^{12}$ \\ Stuart H. Orkin, ${ }^{1,4,8,9}$ Piotr Sicinski, ${ }^{13,14}$ Eric S. Lander, ${ }^{1,15,16,19,20}$ and Harvey F. Lodish ${ }^{1,2,16,19,20}$ \\ ${ }^{1}$ Broad Institute, Cambridge, Massachusetts 02142, USA; ${ }^{2}$ Whitehead Institute for Biomedical Research, Cambridge, \\ Massachusetts 02142, USA; ${ }^{3}$ Department of Medicine, Children's Hospital Boston, Boston, Massachusetts 02115, USA; \\ ${ }^{4}$ Department of Pediatrics, Harvard Medical School, Boston, Massachusetts 02115, USA; ${ }^{5}$ Institute for Chemistry and \\ Biochemistry, Freie Universität Berlin, 14195 Berlin, Germany; ${ }^{6}$ Charité-Universitätsmedizin Berlin, Berlin 10117, Germany; \\ ${ }^{7}$ Center for Molecular Oncologic Pathology, Dana-Farber Cancer Institute, Boston, Massachusetts 02215, USA; ${ }^{8}$ Division of \\ Hematology/Oncology, Children's Hospital Boston, Boston, Massachusetts 02115, USA; ${ }^{9}$ Department of Pediatric Oncology, \\ Dana-Farber Cancer Institute, Boston, Massachusetts, USA; ${ }^{10}$ Institut für Klinische Chemie und Pathobiochemie, Klinikum \\ rechts der Isar, Technische Universität München, 81675 Munich, Germany; ${ }^{11}$ Department of Pathology, Brigham and Women's \\ Hospital, Boston, Massachusetts 02115, USA; ${ }^{12}$ Department of Pathology, Stanford University School of Medicine, Stanford, \\ California 94304, USA; ${ }^{13}$ Department of Cancer Biology, Dana-Farber Cancer Institute, Boston, Massachusetts 02215, USA. \\ ${ }^{14}$ Department of Genetics, ${ }^{15}$ Department of Systems Biology, Harvard Medical School, Boston, Massachusetts 02115, USA. \\ ${ }^{16}$ Department of Biology, Massachusetts Institute of Technology, Cambridge, Massachusetts 02139, USA
}

Genome-wide association studies (GWASs) have identified a genetic variant of moderate effect size at 6p21.1 associated with erythrocyte traits in humans. We show that this variant affects an erythroid-specific enhancer of CCND3. A Ccnd3 knockout mouse phenocopies these erythroid phenotypes, with a dramatic increase in erythrocyte size and a concomitant decrease in erythrocyte number. By examining human and mouse primary erythroid cells, we demonstrate that the CCND3 gene product cyclin D3 regulates the number of cell divisions that erythroid precursors undergo during terminal differentiation, thereby controlling erythrocyte size and number. We illustrate how cell type-specific specialization can occur for general cell cycle components-a finding resulting from the biological follow-up of unbiased human genetic studies.

[Keywords: cell cycle; cell division; cyclin D3; erythrocyte; erythropoiesis; genome-wide association study; human genetics]

Supplemental material is available for this article.

Received May 24, 2012; revised version accepted July 26, 2012.

Genome-wide association studies (GWASs) have identified hundreds of loci associated with numerous phenotypes and diseases in humans (Manolio et al. 2008; Lander 2011). The description of these loci holds promise for the identification of important biological pathways and disease mechanisms. However, while there are notable exceptions (Sankaran et al. 2008a; Musunuru et al. 2010; Zhang et al. 2011), the majority of such molecular

\footnotetext{
${ }^{17}$ These authors contributed equally to this work.

${ }^{18}$ These authors contributed equally to this work.

${ }^{19}$ These authors contributed equally to this work.

${ }^{20}$ Corresponding authors

E-mail lodish@wi.mit.edu

E-mail lander@broadinstitute.org

Article published online ahead of print. Article and pubication date are online at http://www.genesdev.org/cgi/doi/10.1101/gad.197020.112.
}

pathways have yet to be elucidated (Lander 2011). Disorders affecting red blood cells (RBCs) or erythrocytes are among the most common genetic conditions in humans (Balarajan et al. 2011). This includes mutations affecting the globin genes and other erythrocyte components, such as the enzyme glucose-6-phosphate dehydrogenase. Anemia is estimated to affect nearly a quarter of the world's population, much of which is attributable to defective erythrocyte production (Balarajan et al. 2011). An increased understanding of the process through which erythrocytes are produced- erythropoiesis-is important in gaining insight into the pathophysiology of these diseases and may lead to avenues for rationally intervening in these conditions.

A number of recently published GWASs have uncovered variants that affect several erythrocyte traits (Ferreira 
et al. 2009; Ganesh et al. 2009; Soranzo et al. 2009; Kamatani et al. 2010). Principal among these has been the number of RBCs (RBC count) and the size of these cells (mean corpuscular volume [MCV]), which show natural variation in humans and are both heritable traits. Moreover, variation in these traits is also characteristic of specific pathologic conditions affecting erythropoiesis. For example, the megaloblastic anemias and certain congenital anemias are characterized by a markedly elevated MCV. In addition, evolutionary biologists have noted for centuries that the two traits show extensive inverse variation across species (Boylan et al. 1991; Hawkey et al. 1991). The GWASs revealed a few loci that, in an analogous fashion, are inversely associated with RBC count and MCV in humans (Ganesh et al. 2009; Soranzo et al. 2009). One such locus is within the gene CCND3.

The CCND3 gene encodes cyclin D3, one of three wellcharacterized members of the D cyclin family that play critical roles as core members of the mammalian cell cycle machinery (Sicinska et al. 2003; Sherr and Roberts 2004; Malumbres and Barbacid 2009). Once induced, D cyclins bind to and activate the cyclin-dependent kinases (CDKs) 4 and 6, which can then phosphorylate the retinoblastoma tumor suppressor gene product (RB1) and RB1-related p107 and p130 proteins (Malumbres and Barbacid 2009). This phosphorylation in turn leads to release or derepression of E2F transcription factors that promote progression from the $\mathrm{G} 1$ to $\mathrm{S}$ phase of the cell cycle (Sherr and Roberts 2004; Malumbres and Barbacid 2009). Among the D cyclins, cyclin D3 is one of the least well studied. It has been suggested to have a role in certain cancers, particularly those of hematopoietic origin (Sicinska et al. 2003; Metcalf et al. 2010). Indeed, rearrangements involving the CCND3 gene have been seen in cases of multiple myeloma and diffuse large cell lymphoma. Mice with a knockout of the Ccnd3 gene have defects in T-lymphocyte development (Sicinska et al. 2003), B-lymphocyte maturation (Cooper et al. 2006), and neutrophil production (Sicinska et al. 2006). Although $\mathrm{Ccnd2} 2^{-1-} ; \mathrm{Ccnd}^{-1-}$ mice have been noted to be severely anemic and die in mid-gestation /Ciemerych et al. 2002), the role of cyclin D3 in erythropoiesis has not been previously explored.

Here we used insight from human genetics to study a variant near the $C C N D 3$ gene that shows an association of moderate effect size with both MCV and RBC count. We show that this variant affects an erythroid-specific enhancer element that regulates CCND3 expression; individuals with the weaker enhancer variant have larger but fewer red cells in their blood. We then used mouse genetics to show that the loss of Ccnd3 results in greatly enlarged erythrocyte size with a concomitant decrease in RBC count, consistent with the findings from the human genetic association studies. We explore the mechanism underlying this phenotype in mouse and human primary cells and show that cyclin D3 plays a critical role in regulating the number of cell divisions that erythroid precursors will undergo before production of a mature erythrocyte, thus controlling erythrocyte size and num- ber. This unexpected link between the cell cycle, erythroid differentiation, and the regulation of erythrocyte production was only uncovered by pursuing the biology underlying the results from unbiased studies of human genetic variation.

\section{Results}

A variant near CCND3 affects an erythroid-specific enhancer element

In GWASs from the CHARGE and HaemGen consortia, the single-nucleotide polymorphism (SNP) most significantly associated with erythrocyte traits, including MCV and $\mathrm{RBC}$ count, was rs9349205 in the CCND3 locus at 6p21.1 ( $P=1.1 \times 10^{-31}$ for MCV) (Ganesh et al. 2009). Imputation of SNPs in a Japanese cohort study demonstrated that this same SNP was also the most significantly associated with MCV and RBC count in this population $\left(P=6.2 \times 10^{-29}\right.$ for MCV) (Kamatani et al. 2010). Given the significant associations seen with this SNP, we examined all SNPs in Caucasian (CEU) and Asian (CHB and JPT) populations from the 1000 Genomes Project Consortium (2010) that were in close linkage disequilibrium $\left(r^{2}>0.8\right)$ with rs9349205 (Supplemental Fig. 1; Supplemental Tables 1, 2). We then intersected these two sets of SNPs and found that only four SNPsrs9349205, rs9394841, rs1410492, and rs3218097-were in close linkage disequilibrium in both populations examined (Supplemental Table 3). Of these four SNPs, it appeared unlikely that rs3218097 or rs 1410492 were the causal variants at this locus because rs3218097 is less significantly associated with the erythroid traits than rs9349205 (Kamatani et al. 2010), and rs1410492 is in complete linkage disequilibrium with rs3218097 in both the CEU and CHB/JPT populations. This left the SNPs rs9349205 and rs9394841 as potential causal variants. There may be untyped common variants that were missed in our analysis, but given the coverage of common variants in the 1000 Genomes Project Consortium (2010), we believe that we were relatively comprehensive in evaluating the majority of common genetic variation at this locus (our analysis should provide complete coverage of all variants present in at least 10 of the 120 samples from the CEU population).

To explore potential functional roles of these SNPs, we studied chromatin immunoprecipitation (ChIP) data from human adult primary erythroid cells. We examined the presence of histone 3 Lys 4 monomethylation (H3K4me1), a well-characterized marker of enhancer elements; histone 3 Lys 4 trimethylation (H3K4me3), a marker of active promoter regions; and histone 3 Lys 9 acetylation (H3K9ac), which marks active chromatin (Heintzman et al. 2007); along with the erythroid transcription factors GATA1, TAL1 (also known as SCL), and KLF1 that, in combination, delineate active chromatin and enhancer states (Cheng et al. 2009; Tallack et al. 2010; Wu et al. 2011). In the region surrounding rs 9349205 , we noticed that there was significant enrichment of the H3K4me1 mark as well as the presence of a site of erythroid-specific 
A

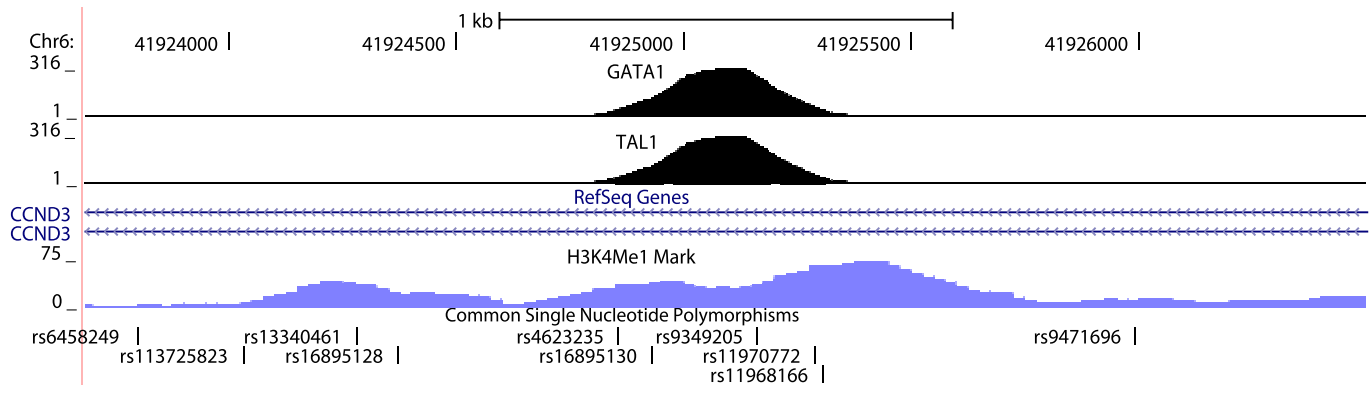

B

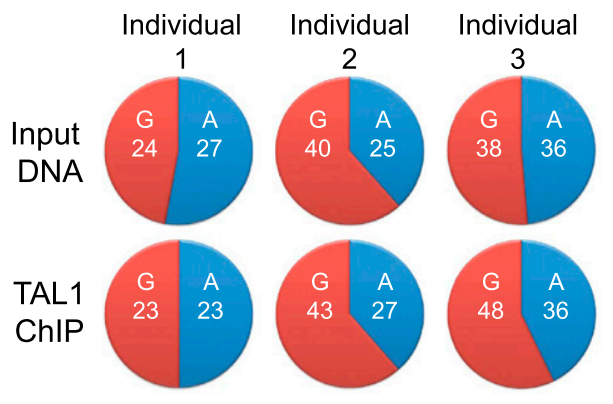

C

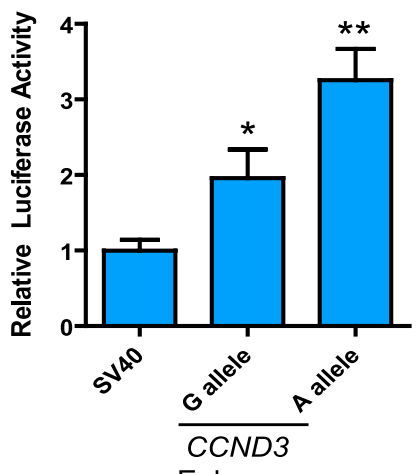

D

293 cells

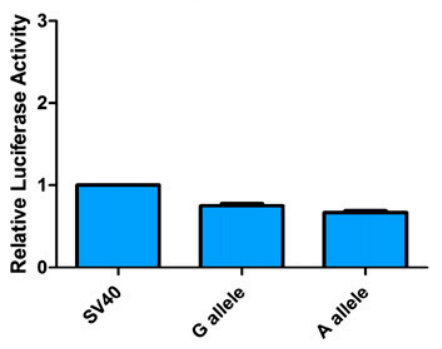

CCND3 Enhancer

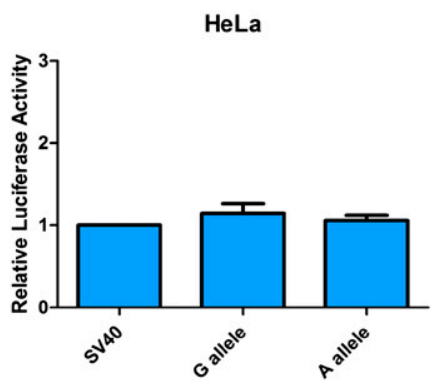

CCND3 Enhancer

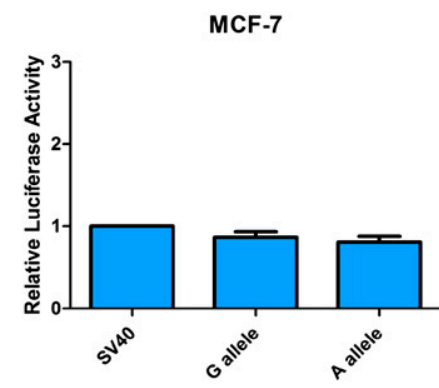

CCND3 Enhancer

Figure 1. GWASs reveal a genetic variant affecting an erythroid-specific enhancer. (A) ChIP-seq (ChIP coupled with deep sequencing) data for GATA1, TAL1, and H3K4me1 are shown in the region surrounding rs9349205, along with other common SNPs in this region. Details of this data are described in the Materials and Methods section. (B) Allele-specific TAL1 ChIP shows no significant differences $(P>0.1$ in all cases) for the number of clones with the G versus A allele at rs9349205. ChIP-PCR products were cloned and then sequenced. The number of clones from input DNA samples and TAL1 ChIP samples are shown in the top and bottom rows of the panel from three separate heterozygous individuals. $(C)$ Results of a luciferase assay using the pGL3-SV40 promoter vector in the absence (indicated by the first bar) or presence of the erythroid-specific 280-bp enhancer region surrounding rs9349205. (Last two bars) The two alleles at rs9349205 were assayed in this experiment. The results are shown as the mean \pm the standard error $(n \geq 3$ per group) and are normalized to Renilla luciferase activity, and the activity of the SV40 promoter construct alone was normalized to a value of $1 .\left(^{\star}\right) P<$ $0.05 ;\left(^{\star *}\right) P<0.01$. (D) The erythroid-specific enhancer region surrounding rs9349205 shows no enhancer activity in human nonerythroid 293, HeLa, and MCF-7 cells. Results of a luciferase assay using the pGL3-SV40 promoter vector in the absence (indicated by the first bar) or presence of the erythroid-specific 280-bp enhancer region surrounding rs9349205. (Last two bars) The two alleles at rs9349205 were assayed in this experiment. The results are shown as the mean \pm the standard error $(n=3$ per group) and are normalized to Renilla luciferase activity, and the activity of the SV40 promoter construct alone was normalized to a value of 1 .

DNase I hypersensitivity, suggesting that this region was a canonical enhancer element (Fig. 1A; Supplemental Figs. 2A, 3A). This enhancer region was found $\sim 15 \mathrm{~kb}$ upstream of the promoter of CCND3, where a variety of regulatory elements of this gene lie. In addition, the erythroid transcription factors TAL1, GATA1, and KLF1 all occupied chromatin in a region surrounding variant rs9349205 in independent ChIP data sets (Fig. 1A; Supplemental Fig. 3A). We noted that rs9349205 was located directly in the middle of the peak of TAL1 and GATA1 chromatin occupancy and was flanked by KLF1-binding peaks. We noted that no such binding sites or signatures 
of regulatory elements were found in the region surrounding variant rs9394841. When we examined the chromatin state in this region from nine human cell lines that were studied as part of the ENCODE project (Ernst et al. 2011), only the erythroid cell line K562 showed signs of having a strong enhancer within this region, whereas the other cell lines did not (Supplemental Fig. 2C). This suggests that this region is an erythroid-specific enhancer element that only shows the appropriate modifications in erythroid lineage cells but not in other cell types.

We explored whether the SNP rs9349205 might directly affect binding by one of the erythroid transcription factors examined above (Fig. 1A; Supplemental Fig. 3A). We focused on GATA1 and TAL1, since the ChIP data suggested that they were bound with their peak centered on rs9349205. We found no consensus transcription factor-binding motifs within the sequence immediately around this SNP (Supplemental Fig. 2B), although a potential noncanonical binding site for TAL1 may exist in the region and would support the finding of a ChIP peak centered at this variant (Fig. 1A; Supplemental Fig. 3A). As a result, we assayed TAL1 binding to chromatin in primary erythroid progenitors cultured from separate individuals heterozygous for the variant at rs9349205 but found no evidence of allele-specific binding in the region containing this SNP (Fig. 1B).

We next sought to address whether this element could functionally act as an enhancer in erythroid cells. A fragment of this region was cloned upstream of a ubiquitous mammalian promoter (SV40) and luciferase construct. When we introduced the 280-base-pair (bp) core region surrounding variant rs9349205, we were able to demonstrate enhancer activity in the K562 erythroid cell line (Fig. 1C). Moreover, when the minor A allele at rs9349205 was introduced into this construct, further increases in activity were noted compared with the $G$ allele (Fig. 1C). This finding supported the hypothesis that the major allele at rs9349205 (G allele), associated with increased erythrocyte size and reduced RBC count, resulted in decreased enhancer activity upstream of the CCND3 gene. Using identical enhancer element assays in several other human nonerythroid cell types, we did not observe any signs of enhancer activity or of any allelespecific differences; these results suggest that rs9349205 affects an erythroid-specific enhancer (Fig. 1D). As noted above, the effect does not appear to be due to differential binding of the TAL1 transcription factor to the sequence surrounding the SNP; the exact mechanisms by which such differential enhancer activity occurs remain to be uncovered. This finding is reminiscent of a well-characterized human mutation in a GATA1-binding site that affects transcriptional activity without affecting GATA1 binding (Martin et al. 1989). We obtained additional evidence supporting the notion that this enhancer element acts specifically on the CCND3 gene; the chromatin conformation capture $(3 \mathrm{C})$ assay demonstrated an increased frequency of interaction of this enhancer element-in comparison with other surrounding regions-with the proximal promoter of CCND3 in human adult erythroid progenitors (Supplemental Fig. 3B). Together, these find- ings support a role of the rs9349205 SNP-most significantly associated with erythroid traits in the $6 \mathrm{p} 21$ region-in altering the erythroid-specific activity of an enhancer element upstream of CCND3.

Given the findings of the erythroid-specific enhancer element of $C C N D 3$, we examined expression of cyclin D3 in samples from in vitro differentiating human adult erythroid progenitors and sections of human bone marrow (Supplemental Fig. 4). We noticed that cyclin D3 was well expressed in early erythroid progenitors, with maximal expression in cells at the proerythroblast stage of differentiation, and underwent a decrease in expression as the cells underwent subsequent terminal differentiation, consistent with findings from prior studies (Dai et al. 2000; Metcalf et al. 2010). The decrease in cyclin D3 expression occurred concomitantly with the period when cell cycle exit occurs during terminal erythropoiesis, suggesting a potential role for this protein in the regulation of this process.

\section{Erythropoiesis in Ccnd3 knockout mice}

To study the in vivo role of $C c n d 3$ in erythropoiesis, we examined a germline knockout mouse of this gene (Sicinska et al. 2003, 2006; Cooper et al. 2006). A mild anemia was present in the adult homozygous knockout mice, with an average $13 \%$ reduction in the hematocrit compared with wild-type animals (Fig. 2A,B). Surprisingly, we noticed a $38 \%$ reduction in RBC count in the mutant animals (Fig. 2C). Along with this decrease in $\mathrm{RBC}$ count, these mice had a dramatic $40 \%$ increase in erythrocyte size (MCV) (Fig. 2D,G,H). To the best of our knowledge, these mice have the largest adult mouse erythrocytes that have been described to date (Fig. $2 \mathrm{G}, \mathrm{H})$. In fact, this $\mathrm{RBC}$ size approaches that seen in normal humans. The erythrocytes in these mice were relatively well hemoglobinized, albeit with a slight decrease in hemoglobin concentration (MCHC [mean corpuscular hemoglobin concentration]) compared to wildtype mice (Fig. 2E). The larger cells have more hemoglobin, and thus fewer RBCs can provide a sufficient total amount of hemoglobin so that little change in the oxygencarrying capacity of these animals would occur. The mice additionally did not show signs of having defective erythropoiesis, as the reticulocyte count as a percent was not significantly different between the groups of mice (Fig. 2F), and when corrected for the RBC count, essentially no difference was observed (an average of $493 \times 10^{3}$ reticulocytes per microliter in controls vs. $488 \times 10^{3}$ reticulocytes per microliter in knockout animals). Other RBC parameters varied concordant with these parameters, and the platelet count showed no change between wild-type and mutant mice (Supplemental Fig. 5A). Of note, the human genetic variant associated with decreased enhancer function (the G allele of rs9349205) is associated with a larger MCV and reduced RBC count, entirely consistent with the phenotype observed in the Ccnd3 knockout animal.

Since erythropoietin (Epo) is a major mitogen and survival factor in erythroid progenitors and D cyclins are often induced by such pathways, we stimulated adult mice with pharmacological doses of Epo. The $C c n d 3^{-/-}$ 

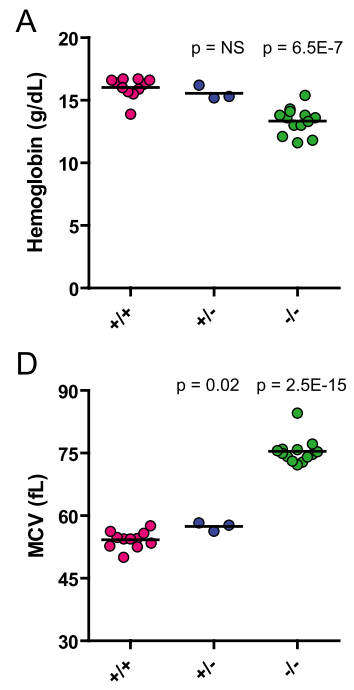

G

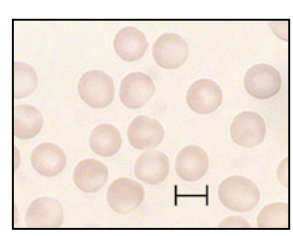

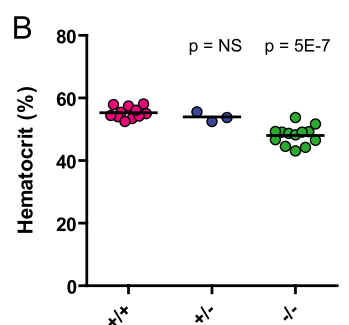

E

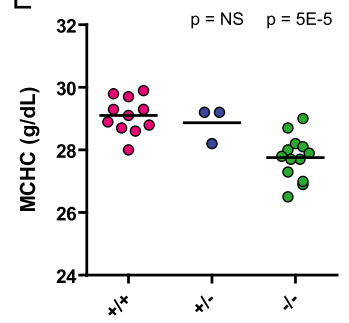

$\mathrm{H}$

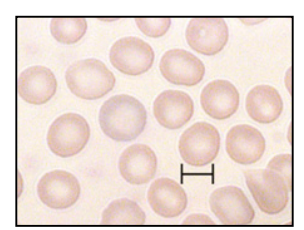

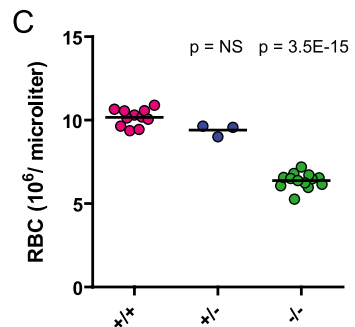

$\mathrm{F}$

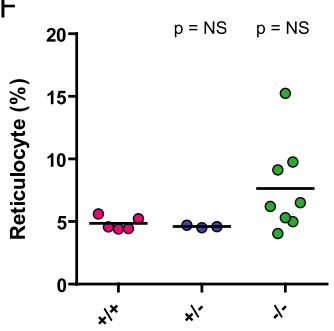

Figure 2. Hematological analysis of Ccnd3 knockout mice reveals a dramatic increase in erythrocyte size with a concomitant decrease in RBC number. $(A-F)$ The hematological values from complete blood counts on mice of various genotypes are shown. The genotype $+/+$ indicates wild-type littermate controls, $+/$ - indicates heterozygous animals, and $-/-$ indicates Ccnd3 knockout animals. The data are shown as the mean, with the distribution of values for $11+/+$, three $+/-$, and $13-/-$ animals, except for the reticulocyte percentage, which was done with five $+/+$, three $+/-$, and eight $-/-$ animals. $P$-values are shown above the corresponding data and are based on a comparison with $+/+$ littermates. $(G, H)$ Blood smears shown with identical $(100 \times)$ magnification demonstrating the enlarged size and heterogeneity of erythrocytes from 8 -wk-old $C \mathrm{cnd} 3^{-/-}$mice $(H)$ in contrast to age-matched littermate $+/+$ controls $(G)$. Bars: $G, H, 5 \mu \mathrm{m}$.

mice continued to respond appropriately to this stimulation despite the absence of cyclin D3, suggesting that cyclin D3 activity was independent of Epo (Supplemental Fig. 6A). Mice with a knockout of the two other D cyclins, Ccnd1 and Ccnd2, were additionally noted to have ostensibly normal erythropoiesis, suggesting that only cyclin D3 had a critical role in terminal erythropoiesis (Supplemental Fig. 5B). This finding is consistent with the patterns of expression seen for these different D cyclins, with cyclin D3 being the predominant protein in terminal erythroid precursor cells (Ciemerych et al. 2002; Metcalf et al. 2010). When the bone marrow in adult mice was examined before and after stimulation with Epo, no major difference among progenitors at various stages of differentiation was noted (Supplemental Fig. 6B). Collectively, these results suggest that, overall, erythropoiesis proceeds in a relatively normal manner, although there is some perturbation in the differentiation process that causes fewer erythrocytes to be produced that are of a much larger size.

\section{Effects of cyclin D3 perturbation on erythroid cells}

To better understand the cellular mechanisms underlying the phenotype seen on loss of Ccnd3 expression, we used in vitro cell culture of primary mouse fetal liver (FL) erythroid cells to examine the mechanistic basis for this alteration in erythrocyte size and number upon knockout or knockdown of Ccnd3 (Zhang et al. 2003). We initially used FL cells derived from mice with a Ccnd3 knockout allele, along with heterozygous and wild-type littermate controls. The cells were labeled with the plasma mem- brane-labeling dye PKH26 in order to study the number of divisions that the cells undergo during differentiation. Normal differentiation proceeds over a period of 2-3 $d$ in such a culture, beginning with undifferentiated erythroid progenitors (Ter119-negative cells from the FL), and generally involves an average of four to five cell divisions before terminal post-mitotic erythroid cells are produced (Zhang et al. 2003). We noticed that there was an average of 0.7 fewer cell divisions at $48 \mathrm{~h}$ in the knockout animals compared with wild-type controls $(P<0.001)$ (Fig. 3B). The cells from the knockout animals maintained brighter expression of $\mathrm{PKH} 26$ as a result of fewer cell divisions occurring between 24 and $48 \mathrm{~h}$ of culture, while maintaining a larger cell size at the 48-h time point, as assessed by forward scatter (Fig. 3A). No major difference was seen in phenotypic markers of erythroid cell differentiation among the knockout or control cultures (Supplemental Fig. 7).

To study the effect of acute knockdown of Ccnd3 and avoid the potential compensatory responses seen in germline knockout cells, we cloned two shRNAs targeting Ccnd3, sh50 and sh79, in mouse retroviral vectors; these shRNAs reduced gene expression to $\sim 15 \%$ and $\sim 40 \%$ of control levels, respectively (Fig. 4A). We found that cells carrying sh50 or sh79 showed no significant difference in the number of divisions after $24 \mathrm{~h}$, but showed significant differences $(P<0.001)$ after $48 \mathrm{~h}$ (Fig. 4B,C). There was an average of $0.9-1.0$ fewer cell divisions with knockdown by the more robust sh50, and 0.2-0.3 fewer cell divisions with knockdown by sh79 (Fig. 4B). Concomitant with this reduction in the number of cell divisions, the cells were larger at $48 \mathrm{~h}$ by measurement of forward scatter (Fig. 4C). 
A
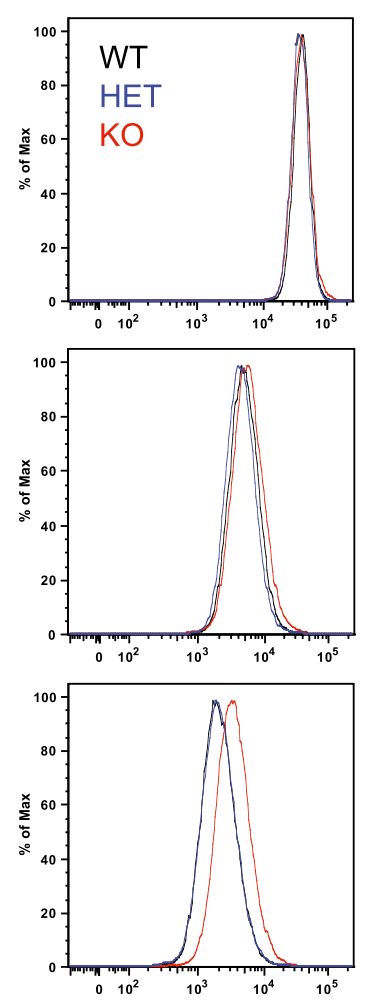

Forward Scatter
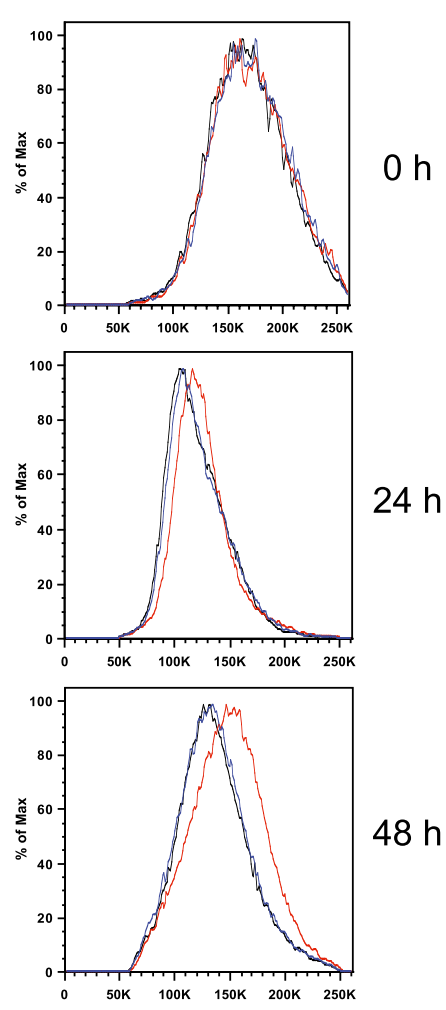

B

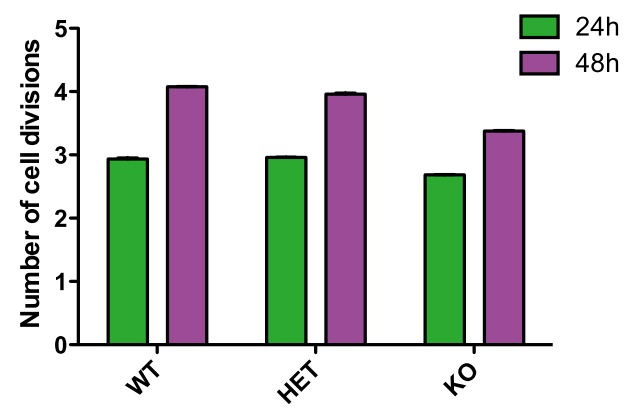

Figure 3. Cyclin D3 regulates the number of cell divisions and cell size during erythropoiesis. (A) PKH26 labeling and forward scatter are shown at 0,24 , and $48 \mathrm{~h}$ for knockout (KO), heterozygous (HET), or wild-type (WT) Ccnd3 FL cells. (B) The average number of cell divisions calculated as discussed in the Materials and Methods from the mean fluorescence intensity measurements for PKH26-labeled FL cells of the various genotypes at 24 and $48 \mathrm{~h}$.

A similar extent of increased size was seen in the subset of enucleated cells examined at $48 \mathrm{~h}$, suggesting that this increased size is maintained in the most mature erythrocytes. Therefore, a reduction in the number of cell divisions occurs upon knockdown of cyclin D3 that results in the production of fewer but larger erythrocytes. The reduction in the number of cell divisions may be attributable to cells that are cycling slower and therefore undergo fewer divisions or may be due to cells that undergo premature cell cycle exit during differentiation.

The finding that only the terminal divisions were affected suggested that the former scenario was less likely to be the case. To directly test this, we labeled the cells at day 1 and day 2 of culture with bromodeoxyuridine (BrdU) and examined cell cycle progression in the cells (Fig. 4D). There was no major difference in the rate of cell cycle progression among the control and knockdown cells (Fig. 4D). Similar to the knockout analysis (Supplemental Fig. 7), we could not detect major differences in the expression of phenotypic markers of erythropoiesis with this knockdown, and enucleation continued to occur, suggesting that differentiation was proceeding in a relatively unperturbed manner. These results strongly suggest that cyclin D3 plays a critical role in regulating the number of cell divisions that occur during terminal erythropoiesis. To ascertain whether this erythroid phenotype was due to interaction between cyclin D3 and CDK4/6, we used the small molecule inhibitor of these kinases, PD0332991 (Toogood et al. 2005). Using a range of concentrations of this inhibitor, known to specifically act on CDK4 and CDK6 (Toogood et al. 2005), we observed identical phenotypes in terms of a reduction in the number of terminal cell divisions and increases in cell size at $48 \mathrm{~h}$ in the same FL cell culture system used in the previous experiments (Supplemental Fig. 8). We additionally studied erythroid cultures from adult bone marrow progenitors and obtained results similar to those discussed above. There was an average reduction of 0.6 cell divisions in cultures from the Ccnd3 knockout mice compared with the controls, although this may be an underestimate of the actual effect on erythropoiesis given the heterogeneity of the progenitor cells cultured from this population (Supplemental Fig. 9). No major differences in cell differentiation, judged by induction of Ter119, or the rate of enucleation were seen when comparing cultures of bone marrow progenitors from Ccnd3 knockout and control mice (Supplemental Fig. 9).

We used knockdown of cyclin D3 in adult human erythroid progenitor cell cultures to further study this phenomenon and discern whether the phenomena observed in the mice were also seen in humans (Sankaran et al. 2008a, 2011). We were able to achieve robust knockdown of cyclin D3 using a set of lentivirally expressed shRNAs (Fig. 5A; Moffat et al. 2006). Consistent with the results from the mouse primary cell culture, we noted that knockdown of CCND3 resulted in a reduction in the number of cell divisions during terminal erythropoiesis, as assayed by PKH26 labeling (Fig. 5B). The distribution of PKH26 labeling was significantly different between the samples $(P<$ 0.001 for all comparisons between knockdown and control samples). Consistent with the results in the mouse primary cell culture system, no differences in cell size or morphology were notable at the early stages of differentiation. However, we did note significant differences in the size of cells during the late stages of differentiation (Fig. 5C; Supplemental Fig. 10). These findings in the human cells-consistent with the data obtained from cultured mouse erythroid cells-reinforce the model that cyclin D3 levels dictate the number of cell divisions that will occur during terminal erythropoiesis, thus regulating the number and size of erythrocyte progeny (Fig. 6). 
A

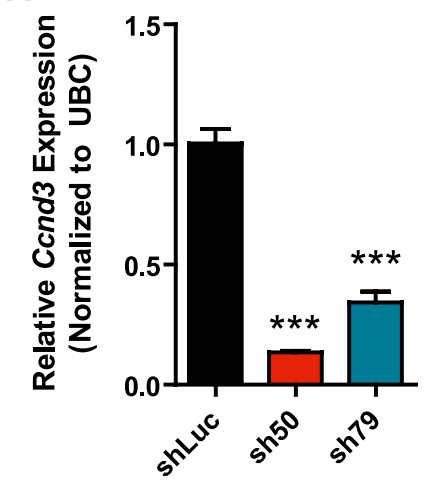

B

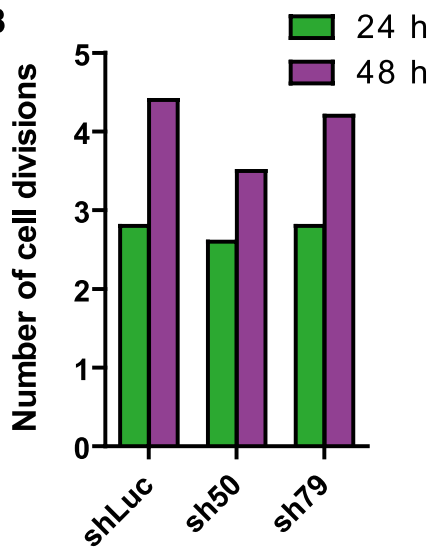

C
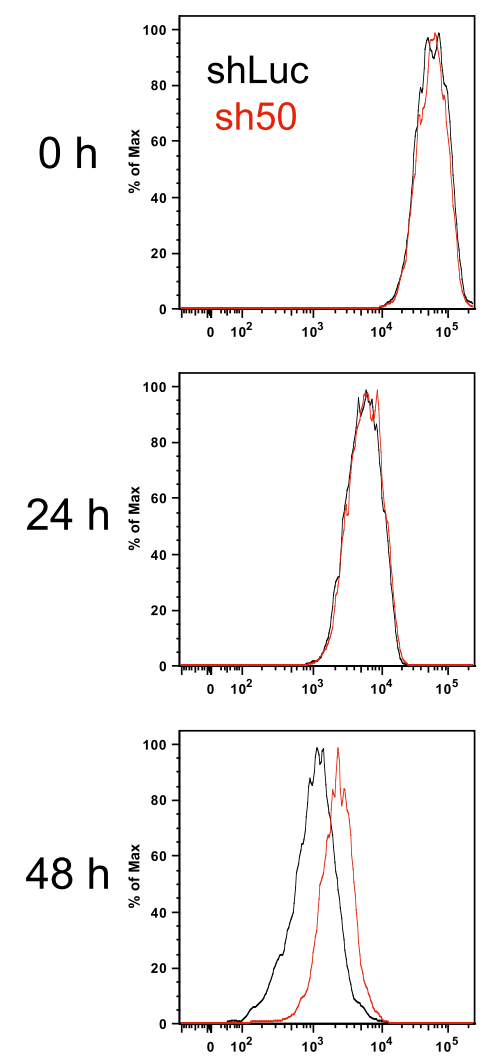

Forward Scatter
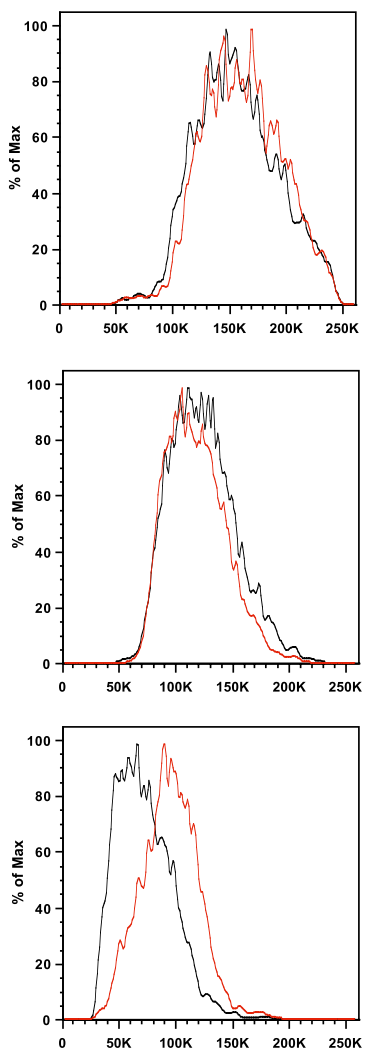

D
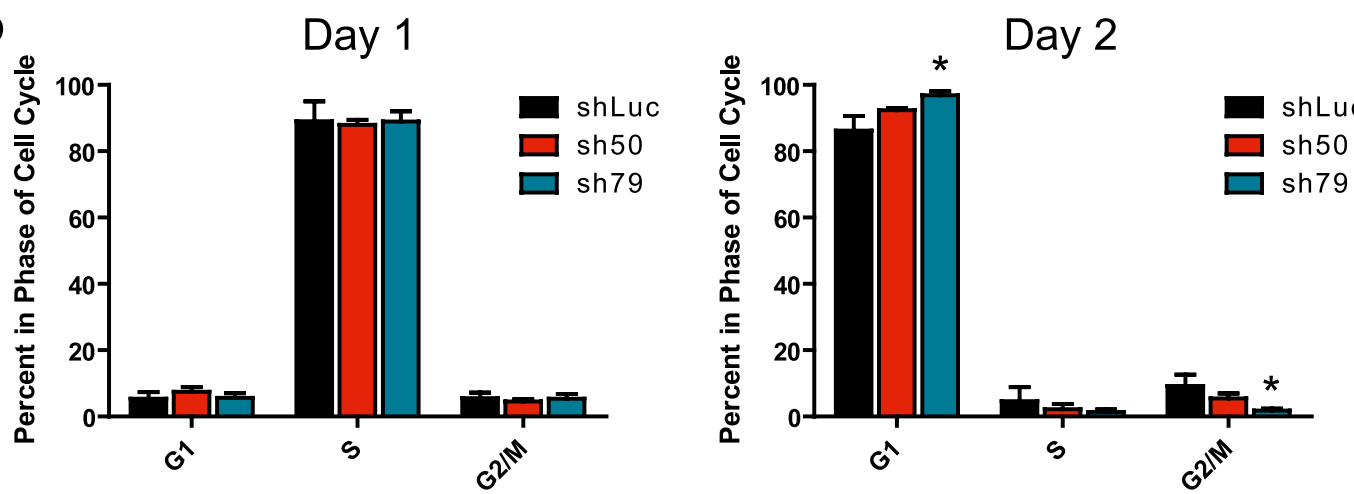

Figure 4. Cyclin D3 regulates the number of cell divisions during terminal mouse erythropoiesis. $(A)$ Relative expression levels of $C c n d 3$ are shown normalized to the $U b c$ control and were measured by quantitative RT-PCR from samples obtained on day 2 of culture. $(B)$ The average number of cell divisions calculated as discussed in the Materials and Methods from the mean fluorescence intensity measurements for PKH26-labeled FL cells (transduced with the luciferase shRNA, shLuc, control, or sh50 and sh79 that target Ccnd3) at 24 and 48 h. (C) An example of the PKH26 labeling, along with forward scatter measurements at 0, 24, and 48 h, is shown for sh50 compared with shLuc. $(D)$ The distribution of cells in various phases of the cell cycle as determined by BrdU labeling for 30 min on days 1 or 2 of culture is shown.

\section{Discussion}

Genetic studies of the metazoan cell cycle machinery have revealed specialized cell type-specific functions for many of the ubiquitously expressed proteins involved, revealing a previously unappreciated extent of complexity for the cell cycle in specialized cell types (Sherr and
Roberts 2004; Malumbres and Barbacid 2009). Our studies on the role of cyclin D3 in erythropoiesis provide an important example of this. In humans, erythroid progenitors need to produce $>2$ million erythrocytes every second by ensuring that maturation and cell cycle exit on terminal differentiation are appropriately coordinated. By following up on clues from human genetic studies, we 
A

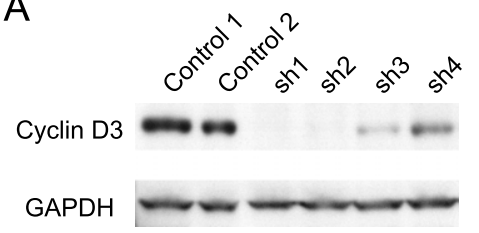

B
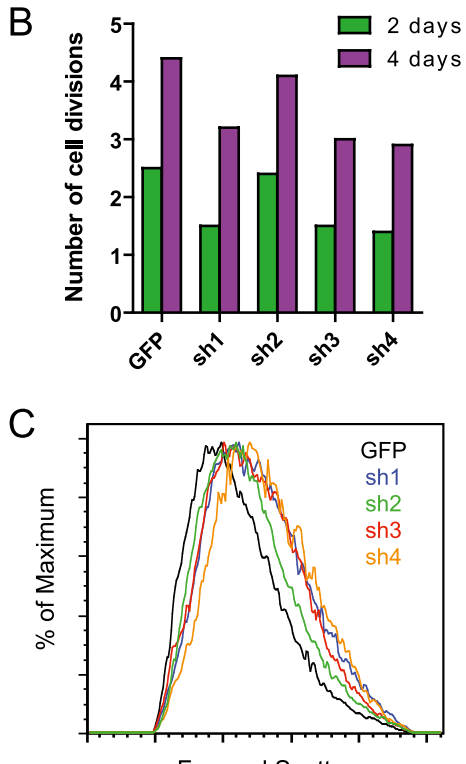

Forward Scatter

Figure 5. Knockdown of cyclin D3 in human erythropoiesis reduces the number of terminal divisions and results in increased cell size. (A) Knockdown of cyclin D3, as evaluated by Western blotting, in K562 cells transduced with the shRNAs targeting CCND3 (sh1-4) or the pLKO.1 control vector (Controls 1 and 2). (B) The average number of terminal divisions is shown for primary adult erythroid cells transduced with sh1-4 or a GFP control lentivirus, which was calculated as discussed in the Materials and Methods from PKH26-labeling data. The cells were labeled on day 2 of differentiation and measured at 2-d intervals. $(C)$ Forward scatter plots are shown for the GFP control or sh1-4 transduced erythroid cells at day 8 of differentiation, at a point when the cells are near the endpoint of terminal maturation.

demonstrated that variation in the expression of cyclin D3 regulates erythrocyte size and number through the coordination of the number of cell divisions that differentiating erythroid progenitors undergo (Fig. 6). Specifically, we showed that the variant rs9349205, which is most significantly associated with $\mathrm{MCV}$ and $\mathrm{RBC}$ count in humans, is located within an erythroid-specific enhancer element of the nearby CCND3 gene. Moreover, the G variant at rs9349205, associated with larger MCV and reduced $\mathrm{RBC}$ count, is associated with decreased activity of this enhancer element, and these individuals therefore would have reduced cyclin D3 levels in developing erythroid progenitors. While we would like to measure the variation in CCND3 mRNA expression in samples from humans with different variants at rs9349205, such experiments are confounded by the variation in both cyclin D3 levels during erythropoiesis and the erythroid differentiation states in different human samples.

Additionally, the effect of variation in erythroid traits by the different alleles at rs9349205 is extremely subtle $10.6 \%$ of the variation in $\mathrm{MCV}$ is due to the allele at rs9349205) (Ganesh et al. 2009), and thus GWASs have required tens of thousands of individuals to delineate such an association with sufficient statistical power. Similarly, one would have to examine the cell cycle in erythroid cells from thousands of individuals with different alleles to reliably detect differences in cell cycle progression specifically due to rs9349205. Given the limitations that exist in following up in humans the subtle phenotypes detected by GWASs, we took the alternative approach of more dramatic perturbations of the candidate genes involved. Indeed, the phenotypes observed in the Cond3 knockout animals and in knockdowns in cultured mouse and human erythroid progenitors are entirely consistent with the human genetic observations; reduced levels of cyclin D3 correlate with fewer terminal erythroid cell divisions, and fewer but larger terminally differentiated cells are produced. Thus, cyclin D3 appears to be a critical regulator of terminal erythroid proliferation through its interaction with CDK4 and CDK6. During terminal erythropoiesis, progenitors undergo progressive reductions in cell size, and premature exit from the cell cycle, triggered by reduced levels of cyclin D3, will therefore produce not only fewer cells, but also cells that are larger in size (Fig. 6).

The molecular mechanisms controlling erythrocyte size and number have been enigmatic despite extensive studies on this topic, particularly regarding the variation in these traits that has occurred in the course of evolution (Boylan et al. 1991; Hawkey et al. 1991; Hoffman 2001). In this study, we demonstrated one mechanism regulating both of these erythrocyte traits. While previous studies suggested that the expression of cell cycle components

Normal
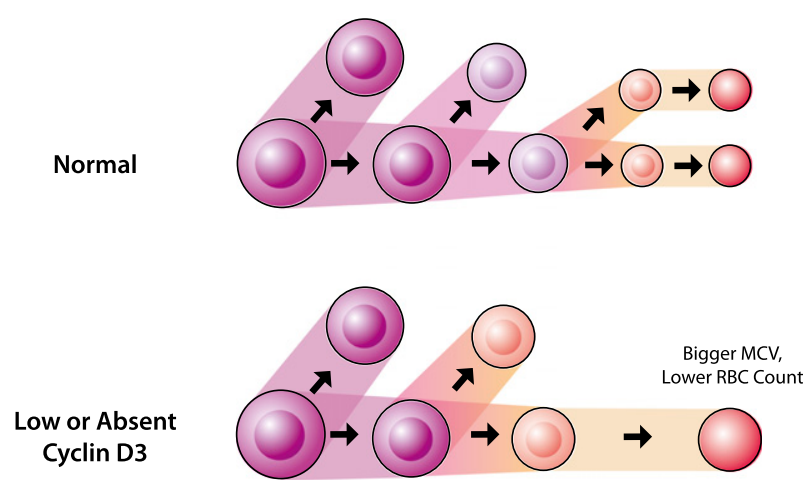

Figure 6. A model of how reduced or absent expression of cyclin D3 can modulate erythropoiesis and cause increased erythrocyte size and reduced RBC counts. The terminal erythroid cells (beginning at the proerythroblast stage of differentiation) undergo a reduced average number of cell divisions during terminal erythropoiesis, and therefore larger erythrocytes are produced with a reduction in the total number of RBCs. This model is supported by the human and mouse genetic data as well as the cellular studies presented here. 
may correlate with changes in size and cell divisions during erythropoiesis (Dolznig et al. 1995), no studies have previously been able to directly examine this functional connection. Over 40 years ago, the number of cell divisions in erythroid precursors was postulated to regulate erythrocyte size, but no examples demonstrating such a connection have previously been described (Stohlman et al. 1968). Our findings show that deregulation of the number of cell divisions in erythroid precursors can have a dramatic impact on both erythrocyte size and number. Further work will be necessary to examine whether variation in the control of cell divisions during erythropoiesis may have given rise to the variations in erythrocyte size and number that have occurred in different species over the course of evolution (Hawkey et al. 1991). The relative preservation of hemoglobin levels seen among different species with inversely varying erythrocyte size and number (Boylan et al. 1991; Hawkey et al. 1991) suggests that such a mechanism coordinately impacting both of these parameters is likely to be involved.

It is interesting to note the agreement between the effects observed with reduction of $C c n d 3$ levels in vitro as compared with an in vivo germline knockout of Ccnd3. We estimate that an average of 0.7 fewer cell divisions occur in the Ccnd3 knockout mice based on the average reduction of 1.6-fold for RBC count in these mice $\left[\log _{2}(1.6)=\right.$ 0.67 , assuming no other alterations in the kinetics of erythropoiesis]. In agreement with this, FL cultures from the Ccnd3 knockout show a reduction of 0.7 fewer cell divisions, and an acutely mediated $\sim 85 \%$ knockdown of Ccnd3 in cultured cells reduced the number of divisions by $\sim 0.9$; these results collectively suggest that if compensatory responses occur in the knockout animals, these are likely to be extremely mild. However, we did note that the extent of knockdown with the lentiviral shRNAs in the K562 cell line did not consistently correlate with the extent of the effect on cell divisions in human primary cells. The exact reasons for this are not currently clear, although this may reflect cell type- or stage-specific variation in activity among various shRNAs. Nonetheless, it is remarkable that we have such overall congruent results from a variety of different experimental and genetic approaches.

Our study demonstrates the power of genetic association studies to provide insight into fundamental biological mechanisms. In this study, we not only took advantage of the results of GWASs to focus on relevant variants that affect human erythropoiesis, we also made use of genomic data to better understand the mechanisms underlying a noncoding genetic variant. Our study reveals the value of such approaches and suggests that even genetic variants with small or moderate effect sizes may provide important insight into basic biological mechanisms of great importance (Musunuru et al. 2010; Lander 2011). The approach that we used to study such subtle human genetic variation will likely be broadly applicable; more dramatic perturbation of genes in loci implicated from GWASs will be needed to study the biology underlying connections uncovered from such genetic studies. Given the large number of loci revealed from GWASs, it is likely that other similarly important biological mechanisms have yet to be unveiled.

\section{Materials and methods}

\section{Cell culture}

293T, HeLa, and MCF-7 cells were maintained in DMEM with $10 \%$ fetal bovine serum (FBS), $2 \mathrm{mM} \mathrm{L-glutamine,} \mathrm{and} 1 \%$ penicillin/streptomycin $(\mathrm{P} / \mathrm{S})$. For MCF-7 cells, the culture medium was additionally supplemented with $10 \mu \mathrm{g} / \mathrm{mL}$ human insulin (Sigma). For production of retro/lentiviruses, 293T cells were transfected with the appropriate viral packaging and genomic vectors using FuGene 6 reagent (Roche) according to the manufacturer's protocol. K562 cells were maintained at a density between $0.1 \times 10^{6}$ and $1 \times 10^{6}$ cells per milliliter in RPMI medium supplemented with 10\% FBS, 2 mM L-glutamine, and $1 \% \mathrm{P} / \mathrm{S}$. Culture of primary human and mouse cells is described below.

\section{Luciferase reporter assay}

K562, 293T, HeLa, and MCF-7 cells were seeded in a 24-well plate at an equal density of 50,000-100,000 cells per well. For transfection of K562 cells in each well, $0.5 \mu \mathrm{g}$ of the luciferase reporter vectors pGL3-SV40 promoter, pGL3-SV40-Enhancer (G allele), or pGL3-SV40-Enhancer (A allele) were cotransfected with $50 \mathrm{ng}$ per well of the pRL-SV40 vector using the Lipofectamine LTX and PLUS transfection reagent according to the manufacturer's protocol (Invitrogen). For transfection of 293T, HeLa, and MCF-7 cells in each well, $0.35 \mu \mathrm{g}$ of the respective luciferase reporter vectors were cotransfected with $35 \mathrm{ng}$ per well of the pRL-SV40 vector using the FuGene 6 reagent according to the manufacturer's protocol (Roche). Cells were incubated at $37^{\circ} \mathrm{C}$ with $5 \% \mathrm{CO}_{2}$ until analysis at $48 \mathrm{~h}$. For measuring luciferase reporter activity, the Dual-Glo Luciferase assay system (Promega) was used according to the manufacturer's protocol. Briefly, cells were resuspended in Dual-Glo Luciferase assay reagent and incubated at room temperature for 10-30 min until measurement of firefly luminescence on a Safire 2 microplate reader (Tecan). Subsequently, Dual-Glo Stop and Glo reagent was added to the suspension and incubated for 10-30 min at room temperature until measurement of Renilla luciferase activity. For each sample, the ratio of Firefly:Renilla luminescence was calculated and normalized to the signal of the pGL3-SV40 promoter control vector.

\section{Constructs}

The shRNA constructs targeting mouse Ccnd3 were sh50 (CCAACCTTCTCAGTTGCCAAAGTCGACTTTGGCAACTG AGAAGGTTGG) and sh79 (CCTTTGCGATGTATCCTCCAT GTCGACATGGAGGATACATCGCAAAGG). These sequences were cloned into the BbsI sites of the MSCV-pgkGFP-U3-U6P vector, which coexpresses GFP from a PGK promoter.

The shRNA constructs targeting human CCND3 were obtained from the Mission shRNA collection (Sigma-Aldrich). The constructs were in the pLKO.1 lentiviral vector. The following were the shRNA sequences that were used: sh1 (CCGGC GCTGTGAGGAGGAAGTCTTCCTCGAGGAAGACTTCCTC CTCACAGCGTTTTT), sh2 (CCGGGCACATGATTTCCTGGC CTTCCTCGAGGAAGGCCAGGAAATCATGTGCTTTTT), sh3 (CCGGCAGACCAGCACTCCTACAGATCTCGAGATCT GTAGGAGTGCTGGTCTGTTTTT), and sh4 (CCGGCCA GCACTCCTACAGATGTCACTCGAGTGACATCTGTAGGA GTGCTGGTTTTT). 
The 280-bp core region surrounding the variant rs9349205 was amplified from human genomic DNA using the Phusion HighFidelity Polymerase (New England Biolabs) and cloned into the NheI and XhoI site of the pGL3-SV40 promoter vector (Promega) to yield pGL3-SV40-Enhancer (G allele). The amplified region was verified by DNA sequencing and was identical to the latest available human genome reference sequence (hg19). The major G allele was mutated to the minor A allele using the QuikChange II XL site-directed mutagenesis kit (Agilent Technologies), according to the manufacturer's protocol, to yield pGL3-SV40-Enhancer (A allele). All constructs were verified by DNA sequencing. The following primers were used for the cloning of the enhancer fragment and mutagenesis: Enhancer-FW (5'-GATCGCTA GCCATTGATCCTTCTGGCCCTA-3'), Enhancer-RV (5'-GATC CTCGAGCTTCAGTCCAAGGACCTGCT-3'), Enhancer-GtoAFW (5'-GCCTTATCACCCACCACTGGCATCCTGCTTC-3'), and Enhancer-GtoA-RV (5'-GAAGCAGGATGCCAGTGGTGGGT GATAAGGC-3').

Mouse FL and bone marrow erythroid progenitor purification, retrovirus infection, in vitro culture, and PD0332991

treatment

Embryonic day 14.5-15.5 (E14.5-E15.5) FL cells were homogenized in PBS supplemented with $2 \%$ FBS and $100 \mu$ M EDTA. Sixweek-old to 12-wk-old mouse bone marrow was harvested in PBS supplemented with $2 \%$ FBS and $100 \mu \mathrm{M}$ EDTA and homogenized, as was done for the FL cells. Mature erythrocytes were lysed by the addition of ammonium chloride solution (Stem Cell Technologies, Inc.) at a 1:4 ratio and incubation for $10 \mathrm{~min}$ on ice. After washing, the remaining cells were incubated with a cocktail of biotin-conjugated antibodies, including Lineage Cocktail (BD Pharmingen, 559971), Ter119 (eBioscience, 13-5921-85), CD16/32 (Abcam, 25249), Sca-1 (BD Pharmingen, 553334), CD34 (MCA,1825B), and CD41 (MCA, 2245B). After magnetic depletion with streptavidin beads (BD Pharmingen, 557812), a pure FL Ter119-negative erythroid progenitor population was obtained (Flygare et al. 2011).

For retroviral infection, 293T cells were transfected with the retroviral construct described above along with the pCL-eco packaging vector. Medium was changed the day after transfection. After $24 \mathrm{~h}$, this medium was collected and filtered at $0.45 \mu \mathrm{m}$ immediately prior to infection of purified erythroid progenitor cells. The cells were mixed with viral supernatant, and polybrene (filtered $4 \mathrm{mg} / \mathrm{mL}$ stock) was added to the mixture at $0.4 \mu \mathrm{L} / \mathrm{mL}$ medium in a 24-well plate at a density of 100,000 cells per well. The cells were spun at $2000 \mathrm{rpm}$ for $90 \mathrm{~min}$ at $32^{\circ} \mathrm{C}$.

Subsequently, for differentiation, cells were resuspended in IMDM containing $15 \% \mathrm{FBS}$ and $0.5 \mathrm{U} / \mathrm{mL}$ Epo (Amgen) for up to $48 \mathrm{~h}$ at $37^{\circ} \mathrm{C}$ with $5 \% \mathrm{CO}_{2}$. Where indicated, cultures were supplemented with 0-2000 nM PD0332991 or DMSO (PD0332991 was kindly supplied by the laboratory of P. Sicinski).

Human $\mathrm{CD} 4^{+}$cell-derived erythroid cultures and lentiviral transduction

Culture of human adult peripheral blood-mobilized CD $34^{+}$progenitors was performed using a two-stage culture method, as has been described previously (Sankaran et al. 2008a, 2011). CD34 ${ }^{+}$ cells were obtained from magnetically sorted mononuclear samples of G-CSF-mobilized peripheral blood from donors and were frozen after isolation. Cells were obtained from the Yale Center of Excellence in Molecular Hematology (YCEMH). Cells were thawed and washed into RPMI-1640 with 10\% FCS, pelleted, and then seeded in StemSpan SFEM medium (Stem Cell Technologies, Inc.) with $1 \times$ CC100 cytokine mix (Stem Cell
Technologies, Inc.) and $2 \% \mathrm{P} / \mathrm{S}$. Cells were maintained in this expansion medium at a density of $0.1 \times 10^{6}-1 \times 10^{6}$ cells per milliliter, with medium changes every other day as necessary. Cells were lentivirally transduced at day 3 of expansion and kept in expansion medium for a total of $6 \mathrm{~d}$. After this expansion phase, the cells were reseeded into StemSpan SFEM medium with $2 \% \mathrm{P} / \mathrm{S}, 20 \mathrm{ng} / \mathrm{mL}$ SCF (PeproTech, Inc.), $1 \mathrm{U} / \mathrm{mL}$ Epo (Amgen), $5 \mathrm{ng} / \mathrm{mL}$ IL-3 (PeproTech, Inc.), $2 \mu \mathrm{M}$ dexamethasone (Sigma-Aldrich), and $1 \mu \mathrm{M} \beta$-estradiol (Sigma-Aldrich). Cells were maintained in differentiation medium, with medium changes every other or every third day as needed. Cells were maintained at a density of $0.1 \times 10^{6}-1 \times 10^{6}$ cells per milliliter. By day 3 of differentiation, homogeneous larger blasts were present in the culture. By day 5, the majority of cells had proerythroblast morphology, and on day 7 , the majority of the cells had basophilic erythroblast morphology. By day 12 of differentiation, the majority of cells demonstrated orthochromatophilic and polychromatophilic erythroblast morphology. This morphological classification has been confirmed previously using phenotypic markers of erythropoiesis, including CD235, CD71, CD45, and CD36.

\section{May-Grünwald-Giemsa staining}

Approximately 50,000-200,000 cells were placed in $200 \mu \mathrm{L}$ of PBS with $1 \%$ FBS and centrifuged onto poly-L-lysine-coated slides by spinning in the cytocentrifugation apparatus for $4 \mathrm{~min}$ at $300 \mathrm{rpm}$. After drying, the slides were stained in MayGrünwald solution (Harleco, Inc.) for $5 \mathrm{~min}$. Slides were washed with water three times ( $30 \mathrm{sec}$ per wash) and stained in Giemsa (Sigma-Aldrich) at a 1:20 dilution for $15 \mathrm{~min}$. Slides were subsequently washed five times (30 sec per wash) with water and dried. Then, slides were mounted with coverslips and examined. All images shown from cytospin sections were taken at $100 \times$ magnification.

\section{Immunohistochemistry}

Immunohistochemistry was performed on sections of normal bone marrow biopsies as has been described previously (Metcalf et al. 2010). Secondary staining with glycophorin A was performed in some samples to detect colocalization of cyclin D3 with erythroid cells and has been shown in a previously published report (Metcalf et al. 2010). Representative sections are shown in the figures.

\section{Analysis of mouse hematopoiesis}

Ccnd3 $3^{-/-}, C c n d 2^{-/-}$, and Ccnd1 $1^{-1-}$ mice have been described previously (Ciemerych et al. 2002; Sicinska et al. 2003, 2006; Kozar et al. 2004; Cooper et al. 2006). Genotyping of these mice was performed as described in the prior publications. The mouse hematopoietic analysis involved performing routine complete blood counts, morphological analysis of peripheral blood and bone marrow, and other methods using standard approaches that have been described in detail before (Sankaran et al. 2008b). Epomediated stimulation of erythropoiesis was carried out in a manner similar to what has been described previously (Singbrant et al. 2011), with daily injections of the Epo over the course of $8 \mathrm{~d}$. All mice were maintained on a $\mathrm{C} 57 \mathrm{Bl} / 6$ background.

FLs were derived from E14.5-E15.5 embryos using timed matings of $\mathrm{C} 57 \mathrm{Bl} / 6$ wild-type and $\mathrm{Ccnd}^{+/-}$mice, as discussed above in the section regarding the isolation of progenitors from mouse FL.

All animal work was performed in accordance with guidelines set forth by the appropriate committees on animal care at the 
Massachusetts Institute of Technology and the Dana-Farber Cancer Institute.

\section{Cell cycle analyses}

In vitro cultured erythroid cells were pulsed with $10 \mu \mathrm{M}$ BrdU for $30 \mathrm{~min}$, and BrdU incorporation was detected using a BrdU flow kit (BD Pharmingen, 552598) at indicated time points. Briefly, pulsed cells were fixed and permeabilized, treated with DNase to expose incorporated BrdU epitopes, and stained by APC-conjugated anti-BrdU antibodies. 7-aminoactinomycin (7-AAD) was added to stain for DNA content. Stained cells were analyzed at a rate of no more than 100 events per second on a BD Pharmingen LSR II flow cytometer. The 7-AAD signal data was acquired on a linear scale.

To allow tracking of the number of cell divisions, FL Ter119negative erythroid progenitor cells from $\mathrm{Ccnd3}^{+/+}, \mathrm{Ccnd3}^{+/-}$, and Ccnd $3^{-/-}$or $\mathrm{C} 57 \mathrm{Bl} / 6$ shRNA-infected cells were labeled with the PKH26 red fluorescent cell linker kit (Sigma-Aldrich, PKH26GL-1KT). Alternatively, lentivirally-transduced human erythroid progenitors were used for this labeling procedure, typically at day 2 of differentiation. Briefly, an equal number of cells was washed with PBS and resuspended in Diluent C. The PKH26 dye was prepared immediately prior to staining, and an equal volume of a $4 \mu \mathrm{M}$ solution in Diluent $\mathrm{C}$ was added to the cell suspension and mixed immediately by pipetting. The cells were incubated for $5 \mathrm{~min}$ at room temperature. The staining reaction was stopped by addition of an equal volume of FBS and incubation for $1 \mathrm{~min}$. Subsequently, the labeled cells were washed three times with IMDM with $10 \%$ FBS, resuspended in Epo-containing medium described above, and cultured at $37^{\circ} \mathrm{C}$ with $5 \% \mathrm{CO}_{2}$ until analysis at the indicated time points. An aliquot of the labeled cells was used to measure the mean fluorescence intensity (MFI) of PKH26 immediately after labeling $(0 \mathrm{~h})$.

Assuming an equal distribution of the fluorophore dye PKH26 on the daughter cells after each cell division, we would anticipate a reduction of the PKH26 MFI by the factor 2 with each division. Accordingly, we would anticipate a reduction of $2^{x}$ after $x$ divisions (Eq. 1) after $y$ hours in culture.

$$
\operatorname{MFI}(\mathrm{y} \mathrm{h})=\frac{M F I(0 \mathrm{~h})}{2^{x}}
$$

Therefore, measuring the MFI after $y$ hours of culture would allow one to approximate the number of cell divisions, $x$, as follows (Eqs. 2-5):

$$
\begin{aligned}
2^{x} & =\frac{M F I(0 \mathrm{~h})}{M F I(y \mathrm{~h})} \\
\log \left[2^{\mathrm{X}}\right] & =\log \left[\frac{M F I(0 \mathrm{~h})}{\operatorname{MFI}(\mathrm{y} \mathrm{h})}\right] \\
\mathrm{X} \cdot \log [2] & =\log \left[\frac{M F I(0 \mathrm{~h})}{M F I(\mathrm{y} \mathrm{h})}\right] \\
\mathrm{X} & =\frac{\log \left[\frac{M F I(0 \mathrm{~h})}{\operatorname{MFI}(\mathrm{yh})}\right]}{\log [2]}
\end{aligned}
$$

\section{Flow cytometry analysis and sorting}

For flow cytometry analysis, in vitro cultured erythroid cells were washed in PBS and stained with $7-\mathrm{AAD}$ or $1 \mu \mathrm{g} / \mathrm{mL}$ propidium iodide (PI), 1:100 APC-conjugated Ter119 (eBioscience, 17-5921-83), 1:300 PE-conjugated CD71 (eBioscience, 12-0711-83), and $1 \mu \mathrm{g} / \mathrm{mL}$ Hoechst, followed by FACS analysis (BD Bioscience LSR II flow cytometer) (Ji et al. 2008). Data were analyzed using FlowJo 8.6.9 (TreeStar).

For flow cytometry sorting of $\mathrm{GFP}^{+}$cells, in vitro cultured erythroid cells were washed with PBS and stained with $1 \mu \mathrm{g} / \mathrm{mL}$ PI. GFP-positive/PI-negative cells were sorted on a BD FACSAria cell sorter.

\section{Quantitative RT-PCR}

Isolation of RNA was performed using the miRNeasy minikit (Qiagen). An on-column DNase (Qiagen) digestion was performed according to the manufacturer's instructions. RNA was quantified by a NanoDrop spectrophotometer (Thermo Scientific). Reverse transcription was carried out using the iScript cDNA synthesis kit (Bio-Rad). Real-time PCR was performed using the ABI 7900 Machine Real-Time PCR system and SYBR Green PCR Master Mix (Applied Biosystems). The following primers were used for quantitative RT-PCR: Ccnd3 forward (5'CGAGCCTCCTACTTCCAGTG-3') and reverse (5'-GGACAG GTAGCGATCCAGGT- $\left.3^{\prime}\right)$, and $U b c$ forward $\left(5^{\prime}\right.$-GAGTTCC GTCTGCTGTGTGA-3 $\left.{ }^{\prime}\right)$ and reverse (5'-CCTCCAGGGTGA TGGTCTTA-3').

\section{Western blotting}

Approximately $2.5 \times 10^{6}$ cells were harvested at indicated time points, washed twice in PBS, resuspended in RIPA lysis buffer (50 mM Tris- $\mathrm{HCl}$ at $\mathrm{pH} 7.4,150 \mathrm{mM} \mathrm{NaCl}, 0.1 \%$ SDS, $1 \% \mathrm{NP}-40$, $0.25 \%$ sodium deoxycholate), and incubated for $10 \mathrm{~min}$ on ice. After centrifugation at $14,000 \mathrm{rpm}$ for $5 \mathrm{~min}$ at $4^{\circ} \mathrm{C}$ to remove cellular debris, the remaining supernatant was transferred to a new tube, supplemented with sample buffer, and incubated for $10 \mathrm{~min}$ at $60^{\circ} \mathrm{C}$. Proteins were separated by SDS gel electrophoresis using the NuPAGE Bis-Tris gel system (Invitrogen) and MOPS running buffer under reducing conditions. Subsequently, proteins were transferred onto a nitrocellulose membrane using the NuPAGE transfer buffer (Invitrogen). Membranes were blocked with $5 \%$ milk-PBST for at least $1 \mathrm{~h}$ and probed with cyclin D3 mouse monoclonal antibody (D7; sc-6283, Santa Cruz Biotechnology) at a 1:500 dilution or GAPDH rabbit polyclonal antibody (FL-335; sc-25778, Santa Cruz Biotechnology) at a $1: 1000$ dilution in $2.5 \%$ milk-PBST for $1 \mathrm{~h}$ at room temperature or overnight at $4^{\circ} \mathrm{C}$. Membranes were washed four times with PBST, incubated with sheep anti-mouse or donkey anti-rabbit peroxidase-coupled secondary antibodies (NA931 and NA934, GE Healthcare) at a 1:2000 dilution in $2.5 \%$ milk-PBST for $1 \mathrm{~h}$ at room temperature, washed three times with PBST, and incubated for 2 min with Western Lightning Plus-ECL substrate (Perkin Elmer). Proteins were visualized by exposure to scientific imaging film (Kodak).

\section{ChIP and $3 C$}

ChIP was performed as has been described previously (Xu et al. 2010). Briefly, $0.5 \times 10^{7}-1 \times 10^{7}$ cells per immunoprecipitation were cross-linked with $1 \%$ formaldehyde for $5 \mathrm{~min}$ at room temperature. Chromatin was sonicated in RIPA buffer with $0.3 \mathrm{M} \mathrm{NaCl}$ to $\sim 500 \mathrm{bp}$. Dynabeads Protein A or G (Invitrogen) was used for collection of chromatin. Antibodies were incubated with beads for $3 \mathrm{~h}$ before incubating with sonicated chromatin overnight. Sequencing of ChIP material took place on the HeliScope single-molecule sequencer, with 5 million-10 million 
reads per sample used in the analysis. The following antibodies were used in the ChIP coupled with deep sequencing (ChIP-seq) analyses: H3K4me1 (Abcam, ab8895), H3K4me3 (Millipore, 04745), H3K9ac (Millipore, 06-942), GATA1 (Abcam, ab47490), TAL1 (Santa Cruz Biotechnology, sc-12984), and KLF1 (Abcam, ab49158). 3C was performed on in vitro derived human adult proerythroblasts (day 5 of differentiation), as has been described previously. A bacterial artificial chromosome (clone RP11720D9 from the Children's Hospital Oakland Research Institute [CHORI]) that covered the CCND3 gene and the upstream enhancer region was used as a control for the $3 \mathrm{C}$ reactions.

For allele-specific ChIP assays, ChIP was performed with TAL1 antibody (Santa Cruz Biotechnology, sc-12984), as discussed above, on CD34-derived erythroid cell samples prospectively genotyped as being heterozygous at rs9349205. PCR of ChIP DNA was performed with AmpliTaq Gold DNA Polymerase (Applied Biosystems) and CCND3 forward and reverse primers encompassing rs9349205 (5'-CT TCAGTCCAAGGACCTGCT-3' and 5'-AAGGGAAGAGGCTGG ATTTG-3'). The 191-bp amplicon was cloned into pCR2.1 (Invitrogen TA cloning kit) according to the manufacturer's directions. Sanger sequencing of individual clones was performed with the forward primer (5'-CTTCAGTCCAAGGACCTGCT-3') to determine genotype at rs9329205.

\section{Statistical analysis of experimental data}

Pairwise comparisons of experimental results were performed using either unpaired two-sided Student's $t$-tests for experimental data with appropriate biological replicates (comparing control samples with experimental samples) or the Fisher's exact tests for comparison of allele frequencies for allele-specific ChIP assays that were done by comparing the input DNA control with the corresponding ChIP sample from a single individual.

\section{Human genetic analysis}

The data from GWASs of erythrocyte traits were analyzed, and the most significantly associated SNPs from each GWAS at the 6p21.1 locus were assessed. We used the SNAP tool (http:// www.broadinstitute.org/mpg/snap) (Johnson et al. 2008) or ad hoc scripts to analyze all common genetic variants from the appropriate 1000 Genomes Project Consortium (2010) populations that are in close linkage disequilibrium with the most significantly associated variants. Given the strong genetic evidence from multiple GWASs that support rs9349205 as being the most significantly associated variant at the 6p21.1 locus, we limited our analysis to variants in close linkage disequilibrium with this variant $\left(r^{2} \geq 0.8\right)$. The University of California at Santa Cruz (UCSC) Genome Browser (http://genome.ucsc.edu) was loaded with custom annotation tracks using data derived from ChIP-seq analysis of various histone modifications and transcription factors in human erythroid cells to analyze the presence of such modifications at the site of the genetic variants. Analysis of chromatin state segmentation (Ernst et al. 2011) was assessed using the appropriate tracks from the ENCODE annotations on the UCSC Genome Browser. The chromatin states were defined as described previously (Ernst et al. 2011).

\section{Acknowledgments}

We are grateful to D. Nathan, S. Lux, C. Walkley, J. Hirschhorn, P. Wong, O. Zuk, G. Lettre, P. Thiru, and R. Do for valuable comments and suggestions. We thank T. DiCesare for assistance with illustrations. This work was supported by NIH grant T32 HL007574-30 (to V.G.S.), a MD Fellowship from the Boehringer Ingelheim Fonds (to L.S.L.), NIH grants R01 CA108420 and P01
CA109901 (to P.S.), and NIH grant P01 HL32262 and a research grant from Amgen, Inc. (to H.F.L.).

\section{References}

1000 Genomes Project Consortium. 2010. A map of human genome variation from population-scale sequencing. Nature 467: 1061-1073.

Balarajan Y, Ramakrishnan U, Ozaltin E, Shankar AH, Subramanian SV. 2011. Anaemia in low-income and middleincome countries. Lancet 378: 2123-2135.

Boylan JW, Van Liew JB, Feig PU. 1991. Inverse changes in erythroid cell volume and number regulate the hematocrit in newborn genetically hypertensive rats. Proc Natl Acad Sci 88: 9848-9852.

Cheng Y, Wu W, Kumar SA, Yu D, Deng W, Tripic T, King DC, Chen KB, Zhang Y, Drautz D, et al. 2009. Erythroid GATA1 function revealed by genome-wide analysis of transcription factor occupancy, histone modifications, and mRNA expression. Genome Res 19: 2172-2184.

Ciemerych MA, Kenney AM, Sicinska E, Kalaszczynska I, Bronson RT, Rowitch DH, Gardner H, Sicinski P. 2002. Development of mice expressing a single D-type cyclin. Genes Dev 16: 3277-3289.

Cooper AB, Sawai CM, Sicinska E, Powers SE, Sicinski P, Clark MR, Aifantis I. 2006. A unique function for cyclin D3 in early B cell development. Nat Immunol 7: 489-497.

Dai MS, Mantel CR, Xia ZB, Broxmeyer HE, Lu L. 2000. An expansion phase precedes terminal erythroid differentiation of hematopoietic progenitor cells from cord blood in vitro and is associated with up-regulation of cyclin E and cyclindependent kinase 2. Blood 96: 3985-3987.

Dolznig H, Bartunek P, Nasmyth K, Mullner EW, Beug H. 1995. Terminal differentiation of normal chicken erythroid progenitors: Shortening of G1 correlates with loss of D-cyclin/ cdk4 expression and altered cell size control. Cell Growth Differ 6: 1341-1352.

Ernst J, Kheradpour P, Mikkelsen TS, Shoresh N, Ward LD, Epstein CB, Zhang X, Wang L, Issner R, Coyne M, et al. 2011. Mapping and analysis of chromatin state dynamics in nine human cell types. Nature 473: 43-49.

Ferreira MA, Hottenga JJ, Warrington NM, Medland SE, Willemsen G, Lawrence RW, Gordon S, de Geus EJ, Henders AK, Smit JH, et al. 2009. Sequence variants in three loci influence monocyte counts and erythrocyte volume. Am J Hum Genet 85: 745-749.

Flygare J, Rayon Estrada V, Shin C, Gupta S, Lodish HF. 2011. HIF1 $\alpha$ synergizes with glucocorticoids to promote BFU-E progenitor self-renewal. Blood 117: 3435-3444.

Ganesh SK, Zakai NA, van Rooij FJ, Soranzo N, Smith AV, Nalls MA, Chen MH, Kottgen A, Glazer NL, Dehghan A, et al. 2009. Multiple loci influence erythrocyte phenotypes in the CHARGE Consortium. Nat Genet 41: 1191-1198.

Hawkey CM, Bennett PM, Gascoyne SC, Hart MG, Kirkwood JK. 1991. Erythrocyte size, number and haemoglobin content in vertebrates. Br I Haematol 77: 392-397.

Heintzman ND, Stuart RK, Hon G, Fu Y, Ching CW, Hawkins RD, Barrera LO, Van Calcar S, Qu C, Ching KA, et al. 2007. Distinct and predictive chromatin signatures of transcriptional promoters and enhancers in the human genome. Nat Genet 39: 311-318.

Hoffman JF. 2001. Questions for red blood cell physiologists to ponder in this millenium. Blood Cells Mol Dis 27: 57-61.

Ji P, Jayapal SR, Lodish HF. 2008. Enucleation of cultured mouse fetal erythroblasts requires Rac GTPases and mDia2. Nat Cell Biol 10: 314-321. 
Johnson AD, Handsaker RE, Pulit SL, Nizzari MM, O'Donnell CJ, de Bakker PI. 2008. SNAP: A Web-based tool for identification and annotation of proxy SNPs using HapMap. Bioinformatics 24: 2938-2939.

Kamatani Y, Matsuda K, Okada Y, Kubo M, Hosono N, Daigo Y, Nakamura Y, Kamatani N. 2010. Genome-wide association study of hematological and biochemical traits in a Japanese population. Nat Genet 42: 210-215.

Kozar K, Ciemerych MA, Rebel VI, Shigematsu H, Zagozdzon A, Sicinska E, Geng Y, Yu Q, Bhattacharya S, Bronson RT, et al. 2004. Mouse development and cell proliferation in the absence of D-cyclins. Cell 118: 477-491.

Lander ES. 2011. Initial impact of the sequencing of the human genome. Nature 470: 187-197.

Malumbres M, Barbacid M. 2009. Cell cycle, CDKs and cancer: A changing paradigm. Natl Rev 9: 153-166.

Manolio TA, Brooks LD, Collins FS. 2008. A HapMap harvest of insights into the genetics of common disease. I Clin Invest 118: $1590-1605$.

Martin DI, Tsai SF, Orkin SH. 1989. Increased $\gamma$-globin expression in a nondeletion HPFH mediated by an erythroidspecific DNA-binding factor. Nature 338: 435-438.

Metcalf RA, Zhao S, Anderson MW, Lu ZS, Galperin I, Marinelli RJ, Cherry AM, Lossos IS, Natkunam Y. 2010. Characterization of D-cyclin proteins in hematolymphoid neoplasms: Lack of specificity of cyclin-D2 and D3 expression in lymphoma subtypes. Mod Pathol 23: 420-433.

Moffat J, Grueneberg DA, Yang X, Kim SY, Kloepfer AM, Hinkle G, Piqani B, Eisenhaure TM, Luo B, Grenier JK, et al. 2006. A lentiviral RNAi library for human and mouse genes applied to an arrayed viral high-content screen. Cell 124: 1283-1298.

Musunuru K, Strong A, Frank-Kamenetsky M, Lee NE, Ahfeldt T, Sachs KV, Li X, Li H, Kuperwasser N, Ruda $\mathrm{VM}$, et al. 2010. From noncoding variant to phenotype via SORT1 at the $1 \mathrm{p} 13$ cholesterol locus. Nature 466: 714-719.

Sankaran VG, Menne TF, Xu J, Akie TE, Lettre G, Van Handel B, Mikkola HK, Hirschhorn JN, Cantor AB, Orkin SH. 2008a. Human fetal hemoglobin expression is regulated by the developmental stage-specific repressor BCL11A. Science 322: 1839-1842.

Sankaran VG, Orkin SH, Walkley CR. 2008b. Rb intrinsically promotes erythropoiesis by coupling cell cycle exit with mitochondrial biogenesis. Genes Dev 22: 463-475.

Sankaran VG, Menne TF, Scepanovic D, Vergilio JA, Ji P, Kim J, Thiru P, Orkin SH, Lander ES, Lodish HF. 2011. MicroRNA15a and -16-1 act via MYB to elevate fetal hemoglobin expression in human trisomy 13. Proc Natl Acad Sci 108: 1519-1524.

Sherr CJ, Roberts JM. 2004. Living with or without cyclins and cyclin-dependent kinases. Genes Dev 18: 2699-2711.

Sicinska E, Aifantis I, Le Cam L, Swat W, Borowski C, Yu Q, Ferrando AA, Levin SD, Geng Y, von Boehmer H, et al. 2003. Requirement for cyclin D3 in lymphocyte development and T cell leukemias. Cancer Cell 4: 451-461.

Sicinska E, Lee YM, Gits J, Shigematsu H, Yu Q, Rebel VI, Geng Y, Marshall CJ, Akashi K, Dorfman DM, et al. 2006. Essential role for cyclin D3 in granulocyte colony-stimulating factordriven expansion of neutrophil granulocytes. Mol Cell Biol 26: 8052-8060.

Singbrant S, Russell MR, Jovic T, Liddicoat B, Izon DJ, Purton LE, Sims NA, Martin TJ, Sankaran VG, Walkley CR. 2011. Erythropoietin couples erythropoiesis, B-lymphopoiesis, and bone homeostasis within the bone marrow microenvironment. Blood 117: 5631-5642.
Soranzo N, Spector TD, Mangino M, Kuhnel B, Rendon A, Teumer A, Willenborg C, Wright B, Chen L, Li M, et al. 2009. A genome-wide meta-analysis identifies 22 loci associated with eight hematological parameters in the HaemGen consortium. Nat Genet 41: 1182-1190.

Stohlman F Jr, Ebbe S, Morse B, Howard D, Donovan J. 1968. Regulation of erythropoiesis. XX. Kinetics of red cell production. Ann N Y Acad Sci 149: 156-172.

Tallack MR, Whitington T, Yuen WS, Wainwright EN, Keys JR, Gardiner BB, Nourbakhsh E, Cloonan N, Grimmond SM, Bailey TL, et al. 2010. A global role for KLF1 in erythropoiesis revealed by ChIP-seq in primary erythroid cells. Genome Res 20: 1052-1063.

Toogood PL, Harvey PJ, Repine JT, Sheehan DJ, VanderWel SN, Zhou H, Keller PR, McNamara DJ, Sherry D, Zhu T, et al. 2005. Discovery of a potent and selective inhibitor of cyclindependent kinase 4/6. J Med Chem 48: 2388-2406.

Wu W, Cheng Y, Keller CA, Ernst J, Kumar SA, Mishra T, Morrissey C, Dorman CM, Chen KB, Drautz D, et al. 2011. Dynamics of the epigenetic landscape during erythroid differentiation after GATA1 restoration. Genome Res 21: $1659-1671$.

Xu J, Sankaran VG, Ni M, Menne TF, Puram RV, Kim W, Orkin SH. 2010. Transcriptional silencing of $\gamma$-globin by BCL11A involves long-range interactions and cooperation with SOX6. Genes Dev 24: 783-798.

Zhang J, Socolovsky M, Gross AW, Lodish HF. 2003. Role of Ras signaling in erythroid differentiation of mouse fetal liver cells: Functional analysis by a flow cytometry-based novel culture system. Blood 102: 3938-3946.

Zhang J, Zahir N, Jiang Q, Miliotis H, Heyraud S, Meng X, Dong B, Xie G, Qiu F, Hao Z, et al. 2011. The autoimmune diseaseassociated PTPN22 variant promotes calpain-mediated Lyp/ Pep degradation associated with lymphocyte and dendritic cell hyperresponsiveness. Nat Genet 43: 902-907. 


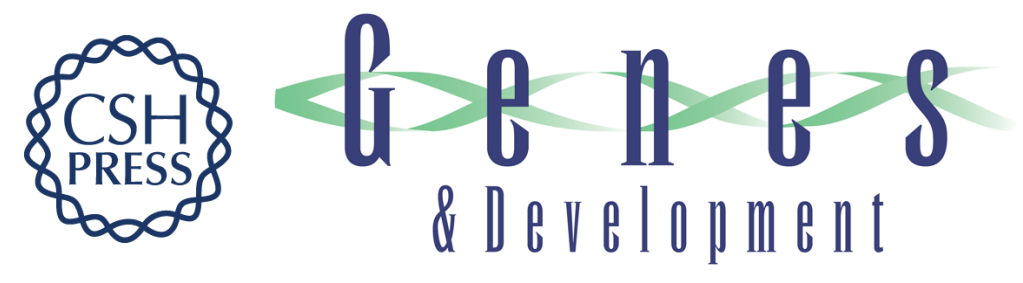

\section{Cyclin D3 coordinates the cell cycle during differentiation to regulate erythrocyte size and number}

Vijay G. Sankaran, Leif S. Ludwig, Ewa Sicinska, et al.

Genes Dev. 2012, 26: originally published online August 28, 2012

Access the most recent version at doi:10.1101/gad.197020.112

\section{Supplemental http://genesdev.cshlp.org/content/suppl/2012/08/22/gad.197020.112.DC1 Material}

References This article cites 41 articles, 16 of which can be accessed free at: http://genesdev.cshlp.org/content/26/18/2075.full.html\#ref-list-1

\section{License}

Email Alerting

Receive free email alerts when new articles cite this article - sign up in the box at the top Service 